\title{
Genome Survey of Misgurnus Anguillicaudatus to Identify Genomic Information, Simple Sequence Repeat (SSR) Markers and Mitochondrial Genome
}

\author{
Guiyun Huang \\ Guangdong Ocean University \\ Jianmeng Cao \\ Chinese Academy of Fishery Sciences Pearl River Fisheries Research Institute \\ Chen Chen \\ Chinese Academy of Fishery Sciences Pearl River Fisheries Research Institute \\ Miao Wang \\ Chinese Academy of Fishery Sciences Pearl River Fisheries Research Institute \\ Zhigang Liu \\ Chinese Academy of Fishery Sciences Pearl River Fisheries Research Institute \\ Fengying Gao \\ Chinese Academy of Fishery Sciences Pearl River Fisheries Research Institute

\section{Mengmeng Yi} \\ Chinese Academy of Fishery Sciences Pearl River Fisheries Research Institute

\section{Gang Chen} \\ Guangdong Ocean University \\ Maixin Lu ( $\nabla$ mx-lu@163.com ) \\ Chinese Academy of Fishery Sciences Pearl River Fisheries Research Institute
}

\section{Research Article}

Keywords: Misgurnus anguillicaudatus, genome characteristics, microsatellite motifs, mitochondrial genome, microsatellite markers

Posted Date: August 20th, 2021

DOI: https://doi.org/10.21203/rs.3.rs-767195/v1

License: (1) This work is licensed under a Creative Commons Attribution 4.0 International License. Read Full License

Version of Record: A version of this preprint was published at Molecular Biology Reports on January 22nd, 2022. See the published version at https://doi.org/10.1007/s11033-021-07037-x. 


\section{Abstract}

The dojo loach Misgurnus anguillicaudatus is an important economic species in Asia because of its nutritional value and broad environmental adaptability. Despite its economic importance, genomic data from $M$. anguillicaudatus was unavailable. In the present study, we conducted a genome survey of $M$. anguillicaudatus using next-generation sequencing technology. Its genome size was estimated to be $1105.97 \mathrm{Mb}$ by using K-mer analysis, and its heterozygosity ratio, repeat sequence content, GC content were $1.45 \%, 58.98 \%$, and $38.03 \%$, respectively. A total of 376,357 microsatellite motifs were identified and mononucleotides, with a frequency of $42.57 \%$, were the most frequently repeated motifs, followed by $40.83 \%$ dinucleotide, $7.49 \%$ trinucleotide, $8.09 \%$ tetranucleotide, and $0.91 \%$ pentanucleotide motifs. The AC/GT, AAT/ATT, and ACAG/CTGT repeats were the most abundant motifs among dinucleotide, trinucleotide, and tetranucleotide motifs, respectively. Besides, a complete mitochondrial genome was sequenced. Based on the maximum likelihood and Bayesian inference analyses, $M$. anguillicaudatus in this study was the "introgressed" mitochondrial type. Furthermore, a total of 376,357 SSR motifs were detected from the genome survey assembly. Seventy microsatellite loci were randomly selected from these SSR loci to test polymorphic, of which, twenty microsatellite loci were assessed in 30 individuals from a wild population. The number of alleles $(\mathrm{Na})$, observed heterozygosity $(\mathrm{Ho})$, and expected heterozygosity $(\mathrm{He})$ per locus ranged from 7 to $19,0.400$ to 0.933 , and 0.752 to 0.938 , respectively. All twenty loci were highly informative $(P I C>0.700)$. Eight loci deviated from Hardy-Weinberg equilibrium after Bonferroni correction $(P<0.05)$. This is the first report of a genome survey in $M$. anguillicaudatus, and genome information, mitochondrial genome, and microsatellite markers will be valuable for further studies on population genetic analysis, natural resource conservation, and molecular marker-assisted selective breeding.

\section{Introduction}

Next-generation, high-throughput sequencing (NGS) is a cost-efficient strategy for generating genomic resources. Genome survey sequencing via NGS provides information on the genome structure of a species, such as the genome size, heterozygosity, and repeat contents. Genome survey sequencing has been widely used to accurately predict the whole genome characteristics of aquaculture species and played an important role in evolutionary biology and adaptation survey [1-5]. In addition to genome information, genome survey sequencing can also generate a large amount of sequence data for the development of molecular markers, such as microsatellites and SNP. Microsatellites (SSRs) are valuable and powerful molecular tools in studies on genetic diversity, determination of parent-offspring relationships, genetic linkage maps, and the identification of quantitative trait loci due to their advantages, such as codominant inheritance patterns, high polymorphism, and random distribution within the genome [68]. Recently, based on genome survey sequencing, many high-quality SSR markers have been developed in aquaculture species such as Charybdis feriatus [9], Sillago sihama [10], Marsupenaeus japonicus [2], and Hemibagrus wyckioides [11].

The dojo loach Misgurnus anguillicaudatus, a member of the family Cobitidae, is an endemic freshwater species in Asia and widely distributed along the eastern coasts of the Asian continent, from the River Amur in China to North Vietnam as well as along the Korean Peninsula, Taiwan, and Japan. M. anguillicaudatus is an important scientific research object due to its unique physiological characteristics. It is regarded as an ideal animal in which to explore the biological origin and evolutionary significance of polyploidization and unisexual reproduction due to its natural variability in ploidy level $[12,13]$. In addition, $M$. anguillicaudatus is also a potential model organism for studies of mechanisms of accessory air-breathing function [14]. Due to its nutritional value and wide environmental adaptability, it has become an important economic species in Asia. Despite its economic importance, dojo loaches for human consumption are mostly obtained through the exploitation of wild populations. Natural populations have declined dramatically because of over exploitation and habitat destruction in recent years. The lack of genetic and genomic resources in $M$. anguillicaudatus has limited the aquaculture production of this species. In the present study, we describe:(1) genome information obtained by genome survey sequence, (2) microsatellite distribution patterns, (3) the complete mitochondrial genome and phylogenetic analysis, (4) the development and validation of polymorphic SSR markers. These results will provide support for natural resource conservation, genetic diversity detection, and molecular marker-assisted selective breeding.

\section{Materials And Methods}

\subsection{Sampling and DNA Extraction}


All experimental procedures for M. anguillicaudatus handling were approved by the Animal Care and Ethics Committee of the Pearl River Fisheries Research Institute, Chinese Academy of Fishery Sciences. Caudal fin samples of $M$. anguillicaudatus were randomly collected from wild populations in Qingyuan City, Guangdong Province, China $\left(24^{\circ} 35^{\prime} 1.311^{\prime \prime} \mathrm{N}, 113^{\circ} 15^{\prime} 86.66^{\prime \prime} \mathrm{E}\right)$. One male adult $M$. anguillicaudatus was chosen as the material for genome survey sequencing. Thirty samples were used to test SSR markers for polymorphisms.

Caudal fins were stored in 95\% ethanol. Total genomic DNA was extracted using the HiPure Mollusk DNA Mini Kit (Magen, China) according to the manufacturer's instructions. The quality and quantity of genomic DNA were assessed by $1.2 \%$ agarose gel electrophoresis, and the concentration was measured on a spectrophotometer (Tiangen, China). Based on the research of Feng et al [15], five EST-SSR markers (Supplementary material Table S1) have been previously applied to identify the ploidy of $M$. anguillicaudatus. The M. anguillicaudatus used for genome survey sequencing was diploid (Supplementary material Figure S1).

\subsection{Whole-Genome Survey Sequencing}

Qualified DNA samples were randomly broken into 350 bp fragments using an ultrasonicator (Covaris Inc.). The TruSeq DNA LT Sample Prep Kit (Illumina, USA) was used to construct libraries. Electrophoresis was used to recover the DNA fragments of required lengths before end repair, following which poly A-tails and sequencing adapters were added. The obtained fragments were purified before PCR amplification for library preparation. The DNA library was sequenced using the Illumina Hiseq Xten platform following the manufacturer's protocol. Library construction and sequencing were performed at the Qingdao OE Biotech Co., Ltd. (Qingdao, China). To ensure the quality of the analysis, we filtered reads that would interfere with subsequent information, reads with adapters, reads with an $\mathrm{N}$ (unable to determine base information) ratio greater than $10 \%$, and low-quality reads from the raw reads to obtain clean reads.

\subsection{Genome Size Estimation and Identification of Heterozygosity and Repeat Ratios}

After removing low-quality reads, all clean data were used for K-mer analysis. Based on the results of the K-mer analysis, information on the peak depth and the number of 17-mers was obtained and used to estimate the size of the genome, repetitive sequences, and heterozygosity. Its relationship was expressed by using the following algorithm: Genome size $=\mathrm{K}$-mer num/Peak depth, where the K-mer num is the total number of K-mers, and the peak depth is the expected value of the K-mer depth [16]. Additionally, the heterozygosity ratio and repeat sequence ratio were estimated following the description in [16]based on K-mer analysis. K-mer analyses were performed using GCE v1.0.0 software.

\subsection{Genome Assembly and GC contents}

The software SOAPdenovo (v2.04) [17] was used for de novo genome assembly. All clean reads were used in the assembly, with a K-mer size of 41 selected as the default parameter to construct a de Bruijn graph. The clean reads were assembled into contigs, and paired-end information was then used to join the unique contigs into scaffolds. The GC content along the assembled sequence was calculated from the proportion of GC out of the total number of bases in the sequencing data.

\subsection{SSR Identification}

The Perl script MIcroSAtellite (MISA, http://pgrc.ipk-gatersleben.de/misa/) was used to identify microsatellite motifs in the de novo draft genome. The search parameters were set for the detection of mono-,di-, tri-, tetra-, penta-, and hexanucleotide microsatellite motifs with a minimum of $10,6,5,5,5$, and 5 repeats, respectively.

\subsection{Mitochondrial genome assembly and phylogenetic analysis}

The filtered clean data were assembled and mapped to complete the mitogenome sequence using NOVOPlasty v3.7.2. with the bait sequence downloaded from GenBank under accession number NC_011209.1.Based on genome sequencing data, only 845bp mitochondrial genome sequences were identified from genome data. Finally, the complete mitochondrial genome of $M$. anguillicaudatus in this study was obtained by next-generation sequencing.

The previously reported mitogenome sequence of 23 species Cobitidae and two outgroup species (Danio rerio and Cyprinus carpio) were downloaded from the GenBank database and used for phylogenetic analyses. The phylogenetic analysis was performed 
based on 13 concatenated mitochondrial protein-coding genes by the Maximum Likelihood (ML) and Bayesian inference (BI) methods, using IQ-TREE and MrBayes 3.1.2 [18], respectively.

\subsection{Characterization of Polymorphic Microsatellite Loci}

Seventy SSR loci containing dinucleotide to pentanucleotide repeats were randomly selected from our genome survey and primers were designed using Primer 5 software to amplify microsatellites. Firstly, seventy SSR loci were tested using ten individuals for polymorphism detection. Then primers of verified polymorphic loci were estimated using a wild population with 30 individuals. The microsatellite polymorphism analysis strategy in this study was performed according to the method described by Schuelke [19]. Briefly, four different universal adapter sequences labeled with the fluorochromes (FAM, VIC, NED, or PET dyes) were added to the 5' end of each forward primer. Universal adapter sequences were shown in Supplementary material Table S2.

PCR was carried out in a $10 \mu \mathrm{L}$ volume that contained $1.0 \mu \mathrm{L}$ of buffer, $0.8 \mu \mathrm{L}$ of dNTP mixture $(2.5 \mathrm{mM}), 0.6 \mu \mathrm{L}$ of Mg${ }^{2+}, 0.05 \mu \mathrm{L}$ of Ex-Taq DNA polymerase, $1 \mu \mathrm{L}$ of DNA (100 ng/ $\mu \mathrm{L}), 4.55 \mu \mathrm{L}$ of $\mathrm{ddH}_{2} \mathrm{O}, 1 \mu \mathrm{L}$ of forward/reverse primer mixture $(\mathrm{F}: \mathrm{R}=2: 50,1.5 \mu \mathrm{M})$, and $1 \mu \mathrm{L}$ of fluorescent-labeled primer $(1.5 \mu \mathrm{M})$. PCR amplification was performed in a thermal lab cycler (SensoQuest, Germany) under the following conditions: initial denaturation at $94^{\circ} \mathrm{C}$ for $5 \mathrm{~min} ; 25$ cycles at $94^{\circ} \mathrm{C}$ for $30 \mathrm{~s}$, appropriate annealing temperature for $1 \mathrm{~min}$, and extension at $72^{\circ} \mathrm{C}$ for $1 \mathrm{~min}$; an additional 12 cycles at $94^{\circ} \mathrm{C}$ for $30 \mathrm{~s}$, annealing at $53^{\circ} \mathrm{C}$ for 1 min, and extension at $72^{\circ} \mathrm{C}$ for $1 \mathrm{~min}$; and a final extension at $72^{\circ} \mathrm{C}$ for $10 \mathrm{~min}$. PCR products were subjected to capillary electrophoresis in a 3730 Genetic Analyzer (Applied Biosystems Inc., Foster City, CA, USA) using GeneScan ${ }^{\text {TM }} 600$ Liz ${ }^{\circledR}$ (Applied Biosystems) as the size standard. The GeneMarker V2.2.0 (ABI) software was used to determine the genotypes.

\subsection{Data analysis}

The number of alleles $\left(N_{a}\right)$, observed heterozygosity $(H o)$, expected heterozygosity $\left(H_{E}\right)$, and polymorphism information content $(P I C)$ of polymorphic loci were calculated using Cervus 3.0.7 software. Tests for deviations from Hardy-Weinberg equilibrium (HWE) were performed using Genepop 4.0. The frequency of null alleles (Fua) was calculated by software Micro-Checker v.2.2.3.

\section{Results}

\subsection{Genome Sequencing and Sequence Quality Estimation}

A total of $145.46 \mathrm{~Gb}$ of raw data were generated by sequencing the genome survey library with $350 \mathrm{bp}$ inserts. After filtering and elimination, $135.22 \mathrm{~Gb}$ of high-quality clean sequences were obtained with Q30 scores assigned to $93.17 \%$, which was approximately $122.3 \times$ coverage. The effective rate and the error rate were $92.96 \%$ and $7 \%$, respectively (Table 1 ).

Table 1

Sequencing data statistics and quality assessment

\begin{tabular}{|llllllll|}
\hline Library & $\begin{array}{l}\text { Insert Size } \\
\text { (bp) }\end{array}$ & $\begin{array}{l}\text { Raw bases } \\
\text { (G) }\end{array}$ & $\begin{array}{l}\text { Clean bases } \\
(\mathbf{G})\end{array}$ & $\begin{array}{l}\text { Effective Rate } \\
(\%)\end{array}$ & $\begin{array}{l}\text { Error Rate } \\
(\%)\end{array}$ & $\begin{array}{l}\text { Q30 } \\
(\%)\end{array}$ & $\begin{array}{l}\text { GC Content } \\
(\%)\end{array}$ \\
\hline $\begin{array}{l}\text { M. } \\
\text { anguillicaudatus }\end{array}$ & 350 & 145.46 & 135.22 & 92.96 & 7 & 95.34 & 38.69 \\
\hline
\end{tabular}

\subsection{Genome Size Prediction and Sequence Assembly}

A total of $135.22 \mathrm{~Gb}$ were used for K-mer analysis in $M$. anguillicaudatus. The 17-mer frequency distribution derived from the sequencing reads was plotted in Figure.1A. The peak of the 17-mer distribution was 90, and the total K-mer count was $99,537,205,022$; therefore, the genome size of $M$. anguillicaudatus was estimated to be $1105.97 \mathrm{Mb}$. The average GC content of the M. anguillicaudatus genome was $38.03 \%$ (Figure.1B). The 17-mer analysis showed that the heterozygosity and repeat content of the $M$. anguillicaudatus genome was $1.45 \%$ and $58.98 \%$, respectively (Table 2 ). 
Table 2

Data statistics and analysis of K-mer $=17 \mathrm{bp}$

\begin{tabular}{|lllllll|}
\hline Sample & $\begin{array}{l}\text { K- } \\
\text { mer }\end{array}$ & $\begin{array}{l}\text { K-mer } \\
\text { Depth }\end{array}$ & $\begin{array}{l}\text { K-mer } \\
\text { Number }\end{array}$ & $\begin{array}{l}\text { Genome } \\
\text { Size(M) }\end{array}$ & $\begin{array}{l}\text { Revised Genome } \\
\text { Size(M) }\end{array}$ & $\begin{array}{l}\text { Heterozygous } \\
\text { Rate (\%) }\end{array}$ \\
\hline $\begin{array}{l}\text { M. } \\
\text { anguillicaudatus }\end{array}$ & 17 & 90 & 99537205022 & 1105.97 & 1099.67 & $\begin{array}{l}\text { Repeat } \\
\text { Rate (\%) }\end{array}$ \\
\hline
\end{tabular}

The assembly statistics of the genome survey sequencing data were summarized in Table 3. A total of 4,120,965 contigs were assembled from the genome survey sequencing data with an N50 of $378 \mathrm{bp}$. Based on the contigs, the genome assembly contained 3,488,543 scaffolds, with an N50 of $551 \mathrm{bp}$.

Table 3

Statistics of the assembled contigs and scaffolds in $M$. anguillicaudatus

\begin{tabular}{|lll|}
\hline Description & Contigs Data & Scaffold Data \\
\hline Total length (bp) & $1,110,202,680$ & $1,176,790,755$ \\
\hline Number of sequences & $4,120,965$ & $3,488,543$ \\
\hline N50 length (bp) & 378 & 551 \\
\hline N60 length (bp) & 289 & 389 \\
\hline N70 length (bp) & 200 & 279 \\
\hline N80 length (bp) & 147 & 172 \\
\hline N90 length (bp) & 118 & 128 \\
\hline Number of N50 & 712341 & 484,744 \\
\hline Number of N60 & 1048358 & 739,119 \\
\hline Number of N70 & 1508194 & 109,574 \\
\hline Number of N80 & 2170190 & 164,087 \\
\hline Number of N90 & 3018783 & 244,573 \\
\hline
\end{tabular}

\subsection{Identification and Characteristics of Microsatellite Motifs in Genome Surveys}

Based on the assembled draft genome sequences, a total of 376,357 microsatellite motifs (SSRs) were identified from the genome, therefore, the microsatellite distribution frequency in the genome was estimated to be approximately 340.3 microsatellites per Mb. Among SSRs, mononucleotide repeats (42.57\%) were the most frequent repeat motifs, followed by dinucleotides (40.83\%), trinucleotides $(7.49 \%)$, tetranucleotides $(8.09 \%)$, pentanucleotides $(0.91 \%)$, and hexanucleotides $(0.10 \%)$ (Table 4$)$. Short repetitive motifs were the dominant motifs, especially mono- and di-repeats, which accounted for $80.40 \%$. 
Table 4

Microsatellites characteristics in the genome of $M$. anguillicaudatus.

\begin{tabular}{|lll|}
\hline SSR mining & Total & Percent (\%) \\
\hline Total number of identified SSRs & 376357 & \\
\hline Mono-nucleotides repeat & 160226 & $42.57 \%$ \\
\hline Di-nucleotides repeat & 153665 & $40.83 \%$ \\
\hline Tri-nucleotides repeat & 28191 & $7.49 \%$ \\
\hline Tetra-nucleotides repeat & 30445 & $8.09 \%$ \\
\hline Penta-nucleotides repeat & 3438 & $0.91 \%$ \\
\hline Hexa-nucleotides repeat & 392 & $0.10 \%$ \\
\hline
\end{tabular}

The number of penta- and hexanucleotide motif types was greater than 83, while that of tri- and tetranucleotides was less than 32 (Figure.2A). The penta- and hexanucleotide SSR loci contained more motif types, each in a relatively small percentage. The frequency distribution range of microsatellite repeats ranged from 5 to 10 repeats for trinucleotides, mostly from 5 to 9 repeats for tetranucleotides, from 5 to 8 repeats for pentanucleotides, and from 5 to 7 repeats for hexanucleotides (Figure.2B). Of tri-, tetra-, penta-, and hexanucleotide repeats, the most common repeat numbers (5-6) accounted for more than $50 \%$ of the total repeats, and the repeat numbers 5-7 accounted for more than $78 \%$ of the total repeats. The motif types increased with increasing repeat length, and the frequency of repeats decreased because mutation rates were higher in longer repeats.

In dinucleotide SSR loci, AC/GT (46.52\%) was the most abundant repeat motif, and CG/CG was the least abundant (0.32\%) in Figure.3A. Of the trinucleotide repeats, AAT/ATT was the most abundant trinucleotide motif, accounting for $64.01 \%$, followed by ATC/ATG (10.27\%) and AAC/GTT (9.53\%), as shown in Figure.3B. Of the tetranucleotide repeats, ACAG/CTGT was the most abundant motif (34.02\%), followed by AGAT/ATCT (12.83\%) and AAAT/ATTT (10.64\%) (Supplementary material Table S3).

\subsection{Mitochondrial genome assembly and phylogenetic analysis}

The $M$. anguillicaudatus mitochondrial genome formed a closed circular molecule with a total length of $16,646 \mathrm{bp}$ in size. It consisted of 13 protein coding genes, 22 tRNA genes, 2 rRNA genes, and a non-coding hypervariable control region showing the typical teleosts mitogenomic arrangement (Figure.4). The mitochondrial genome was AT-biased (58.05\%) with the lowest frequency for $\mathrm{G}$ among the four bases and an $\mathrm{A}+\mathrm{T}$ rich pattern, which was in accordance with the characteristics of the mitochondrial genomes of vertebrates. Except for one protein coding gene (ND6) and eight tRNAs (tRNA-Ala, tRNA-Asn, tRNA-Cys, tRNA-Tyr, tRNA-Ser, tRNA-Glu, tRNA-Pro, tRNA-GIn) encoded on the light strand, all other genes were encoded on the heavy strand. In the complete mitogenome, eleven intergenic spacers were observed: of these, nine intergenic spacers were small intergenic spacers ranging from 1 to $13 \mathrm{bp}$ with a total length of $32 \mathrm{bp}$. The largest two intergenic spacers were found in tRNA-Asn/tRNA-Cys (30 bp) occurring in the light strand and COX2/tRNA-Lys (27 bp) occurring in the heavy strand. A total of six overlapping regions were identified in a total of $26 \mathrm{bp}$ in length. Gene overlaps were observed at 11 gene junctions (tRNA-lle/tRNA-GIn, ATP8/ATP6, ATP6/COX3, Nd4L/ND4, ND5/ND6, tRNA-Thr/tRNA-Pro), with overlaps ranging from 1 to $10 \mathrm{bp}$. Regarding start and stop codons, except for COX1 starting with GTG, the remaining 11 protein coding genes start with ATG. It is important to note that some of the protein coding genes (2 of 13 genes) are inferred to terminate with an incomplete stop codon (COX3, CYTB), with four (ND2, ND3, ND4, ND5) using TAG as a stop codon, and other seven genes (ND1, COX1, COX2, ATP8, ATP6, ND4L, ND6) sharing TAA, respectively (Supplementary material S4).

To determine the taxonomic status of $M$. anguillicaudatus, we performed the phylogenetic relationship of this $M$. anguillicaudatus with other Cobitidae species as inferred by the entire mitogenome. The phylogenetic analyses trees constructed by $\mathrm{ML}$ and $\mathrm{BI}$ methods provided identical phylogenetic topologies (Figure. 5). All nodes in the ML and BI tree were strongly supported. With Cyprinus carpio and Danio rerio as outgroups, the species from Cobitinae, Nemacheilinae, and Botiinae were monophyletic groups, then form one clade. However, within the Cobitinae, the genus Misgurnus was not in a monophyletic group but divided into two divergent clades. One clade included the M. anguillicaudatus (NC_011209.1) and M. anguillicaudatus (DQ026434.1), and this 
clade clustered with other genus Misgurnus species (M. nikolskyi and M. mohoity). While in the other clade, the $M$.

anguillicaudatus obtained in this study, the M. anguillicaudatus (MF579257.1), and M. anguillicaudatus (HM856629.1) were clustered together, then this clade was closely related to genus Cobitis species, such as Cobits lutheri, C. macrostigma, $C$. sinensis, and C. striata.

\subsection{Polymorphism of Microsatellite Loci}

Out of 70 microsatellite loci, a total of 20 loci showed polymorphism in a wild $M$. anguillicaudatus population of 30 individuals (Table 5). A total of 237 alleles were detected for the 20 SSR markers in the 30 individuals, and the number of alleles $(\mathrm{Na})$ per locus ranged from 7 (MA-SSR-19, MA-SSR-48) to 19 (MA-SSR-17), with an average of 11.85 alleles per locus. The observed heterozygosity $(\mathrm{Ho})$ ranged from 0.400 to 0.933 , and the average Ho was 0.638 . The Ho value of MA-SSR-17 (0.933) was highest among all loci, indicating high heterozygosity of this locus, while the Ho value of MA-SSR-70 (0.400) was lowest in the detected loci. The average expected heterozygosity $(\mathrm{He})$ was 0.877 , ranging from 0.752 to 0.938 , indicating a high degree of genetic variation for these individuals. 
Table 5

Characterization of 20 polymorphic microsatellite loci in $M$. anguillicaudatus.

\begin{tabular}{|c|c|c|c|c|c|c|c|c|c|}
\hline Primers & Sequences $5^{\prime}-3^{\prime}$ & Motifs & $\begin{array}{l}\text { Allele } \\
\text { size } \\
\text { (bp) }\end{array}$ & $\mathrm{Na}$ & Ho & $\mathrm{He}$ & $P I C$ & $P_{H W E}$ & $F($ Null $)$ \\
\hline \multirow[t]{2}{*}{$\begin{array}{l}\text { MA- } \\
\text { SSR-04 }\end{array}$} & F:GAGTCCACGATACACACACAGA & $($ ATAG) 24 & $\begin{array}{l}248- \\
292\end{array}$ & 11 & 0.500 & 0.881 & 0.839 & 0.070 & 0.218 \\
\hline & R:AACATGTTAACTTACCCTGTCT & & & & & & & & \\
\hline \multirow[t]{2}{*}{$\begin{array}{l}\text { MA- } \\
\text { SSR-09 }\end{array}$} & F:TGAAGACCAGGTGTGACTATGT & (GA) 57 & $\begin{array}{l}186- \\
300\end{array}$ & 12 & 0.579 & 0.912 & 0.878 & 0.001 ** & 0.212 \\
\hline & R:TTCCTACCAGCAGATCCAATAC & & & & & & & & \\
\hline \multirow[t]{2}{*}{$\begin{array}{l}\text { MA- } \\
\text { SSR-10 }\end{array}$} & F:TGCCCTATTACTTTGTTACAGT & (CTAT)23 & $\begin{array}{l}170- \\
262\end{array}$ & 13 & 0.524 & 0.925 & 0.894 & $0.000 \star \star \star$ & 0.2668 \\
\hline & R:GTTTCCCATGCAAAACGAG & & & & & & & & \\
\hline \multirow[t]{2}{*}{$\begin{array}{l}\text { MA- } \\
\text { SSR-11 }\end{array}$} & F:GCAAGAGACGCACAGACTTACT & $($ TAGA) 29 & $\begin{array}{l}256- \\
328\end{array}$ & 11 & 0.571 & 0.847 & 0.814 & $0.000 * * *$ & 0.1717 \\
\hline & R:AAGCTTACAGGGTAGGGAATGA & & & & & & & & \\
\hline \multirow[t]{2}{*}{$\begin{array}{l}\text { MA- } \\
\text { SSR-17 }\end{array}$} & F:ACACAGCCTCTTCTTGGTAATA & (GAATT)8 & $\begin{array}{l}185- \\
290\end{array}$ & 19 & 0.933 & 0.938 & 0.917 & 0.093 & 0.0055 \\
\hline & R:TCTCTTTGGCAGAATTGTACCT & & & & & & & & \\
\hline \multirow[t]{2}{*}{$\begin{array}{l}\text { MA- } \\
\text { SSR-19 }\end{array}$} & F:AAATTAACCGAACAAGCCAACT & (ATTCA) 10 & $\begin{array}{l}206- \\
251\end{array}$ & 7 & 0.600 & 0.752 & 0.700 & 0.297 & 0.0681 \\
\hline & R:TGCGCTTTCACTCCTTCTATTA & & & & & & & & \\
\hline \multirow[t]{2}{*}{$\begin{array}{l}\text { MA- } \\
\text { SSR-31 }\end{array}$} & F:GGTTCGTGAAAATGAATCGG & $(\mathrm{AGACC}) 8$ & $\begin{array}{l}195- \\
330\end{array}$ & 10 & 0.478 & 0.753 & 0.702 & 0.113 & 0.2016 \\
\hline & R:CCTCACCTGACСТTCTCTCG & & & & & & & & \\
\hline \multirow[t]{2}{*}{$\begin{array}{l}\text { MA- } \\
\text { SSR-34 }\end{array}$} & F:TGTAGATCCACTGATCAAACTGA & (TATC)9 & $\begin{array}{l}296- \\
372\end{array}$ & 15 & 0.724 & 0.897 & 0.871 & 0.050 & 0.1015 \\
\hline & R:TGAGCTCAATGAACCCATCA & & & & & & & & \\
\hline \multirow[t]{2}{*}{$\begin{array}{l}\text { MA- } \\
\text { SSR-35 }\end{array}$} & F:CGTCATCATTGATCTGCGAC & $($ GATA $) 13$ & $\begin{array}{l}218- \\
290\end{array}$ & 15 & 0.680 & 0.920 & 0.894 & $0.000 * \star \star$ & 0.1401 \\
\hline & R:GTCGGGGTACCCTTTAATGC & & & & & & & & \\
\hline \multirow[t]{2}{*}{$\begin{array}{l}\text { MA- } \\
\text { SSR-38 }\end{array}$} & F:GGGTACTATCCCATTGACAGC & $($ GAAT $) 9$ & $\begin{array}{l}345- \\
393\end{array}$ & 10 & 0.435 & 0.894 & 0.862 & $0.001 * \star$ & 0.335 \\
\hline & R:TTCTGCACAACCTCATGACC & & & & & & & & \\
\hline \multirow[t]{2}{*}{$\begin{array}{l}\text { MA- } \\
\text { SSR-39 }\end{array}$} & F:TGCCAGGACACCATTTATGA & $($ GATA) 9 & $\begin{array}{l}226- \\
282\end{array}$ & 14 & 0.625 & 0.911 & 0.883 & 0.425 & 0.1775 \\
\hline & R:GGGAGAACCGAATCCCTCTA & & & & & & & & \\
\hline
\end{tabular}


Table 5

Continued

\begin{tabular}{|c|c|c|c|c|c|c|c|c|c|}
\hline Primers & Sequences $5^{\prime}-3^{\prime}$ & Motifs & $\begin{array}{l}\text { Allele } \\
\text { size } \\
\text { (bp) }\end{array}$ & $\mathrm{Na}$ & Ho & $\mathrm{He}$ & $P I C$ & $P_{H W E}$ & $F(N u l l)$ \\
\hline \multirow[t]{2}{*}{$\begin{array}{l}\text { MA- } \\
\text { SSR-40 }\end{array}$} & F:ATCACATGCTTCCTCTGGCT & (TGTC)9 & $\begin{array}{l}266 \\
- \\
238\end{array}$ & 11 & 0.882 & 0.870 & 0.831 & 0.877 & -0.018 \\
\hline & R:AAAGCACAAACCCTTAAAGACTG & & & & & & & & \\
\hline \multirow[t]{2}{*}{$\begin{array}{l}\text { MA- } \\
\text { SSR-43 }\end{array}$} & F:CATTTCTCAATGGGACTGGC & $($ TCTA $) 9$ & $\begin{array}{l}237- \\
369\end{array}$ & 12 & 0.667 & 0.899 & 0.850 & $0.016^{\star}$ & 0.1417 \\
\hline & R:GGCCACAAACATCTTTGACA & & & & & & & & \\
\hline \multirow[t]{2}{*}{$\begin{array}{l}\text { MA- } \\
\text { SSR-48 }\end{array}$} & F:GCTTTCTGTGGAGCGAACTC & (ATT)12 & $\begin{array}{l}255- \\
276\end{array}$ & 7 & 0.818 & 0.848 & 0.784 & 0.137 & 0.0109 \\
\hline & R:AGCCCTGCATTTGTTAGAGG & & & & & & & & \\
\hline \multirow{2}{*}{$\begin{array}{l}\text { MA- } \\
\text { SSR- } \\
51\end{array}$} & F:AACGTGGCGTAACAAAGTGT & $($ TAACA $) 8$ & $\begin{array}{l}210- \\
290\end{array}$ & 11 & 0.529 & 0.904 & 0.865 & $0.000 * \star \star$ & 0.2468 \\
\hline & R:TGCCATGTTACGTTGTTATGTT & & & & & & & & \\
\hline \multirow[t]{2}{*}{$\begin{array}{l}\text { MA- } \\
\text { SSR-58 }\end{array}$} & F:CAAACAGACACCCACACACA & $(A C A G) 11$ & $\begin{array}{l}210- \\
390\end{array}$ & 17 & 0.714 & 0.919 & 0.895 & $0.005^{\star \star}$ & 0.1186 \\
\hline & R:CGTCTTCCTCCTCCTCACAG & & & & & & & & \\
\hline \multirow[t]{2}{*}{$\begin{array}{l}\text { MA- } \\
\text { SSR-67 }\end{array}$} & F:TTAGTGAAGGGAGGGTGTGG & (TATTC)7 & $\begin{array}{l}226- \\
291\end{array}$ & 10 & 0.714 & 0.858 & 0.819 & 0.150 & 0.0845 \\
\hline & R:TGCATGATGGACCTTTTGAA & & & & & & & & \\
\hline \multirow[t]{2}{*}{$\begin{array}{l}\text { MA- } \\
\text { SSR-68 }\end{array}$} & F:GCCATGTAGTGTTTGGTCCAT & $($ AAATA $) 7$ & $\begin{array}{l}195- \\
375\end{array}$ & 8 & 0.583 & 0.859 & 0.801 & 0.323 & 0.1718 \\
\hline & R:GTTACGTTTTGCAGGTCCGT & & & & & & & & \\
\hline \multirow[t]{2}{*}{$\begin{array}{l}\text { MA- } \\
\text { SSR-69 }\end{array}$} & F:CGTCACCTGTAACTCTGGGTC & $(A C A G) 10$ & $\begin{array}{l}223- \\
287\end{array}$ & 16 & 0.800 & 0.909 & 0.881 & 0.705 & 0.0505 \\
\hline & R:CAAACAGAATATTGAGGCCG & & & & & & & & \\
\hline \multirow[t]{2}{*}{$\begin{array}{l}\text { MA- } \\
\text { SSR-70 }\end{array}$} & F:TAGGGATAAGCACCGACCTG & $(\mathrm{CTAT}) 14$ & $\begin{array}{l}152- \\
280\end{array}$ & 8 & 0.400 & 0.846 & 0.795 & 0.089 & 0.3467 \\
\hline & R:AACCTTTTTGGCACGTTCAC & & & & & & & & \\
\hline \multicolumn{4}{|l|}{ Mean } & 11.85 & 0.638 & 0.877 & 0.839 & & \\
\hline \multicolumn{10}{|c|}{$\begin{array}{l}\text { Primers for each locus including a forward primer }(\mathrm{F}) \text {, a reverse primer }(\mathrm{R}), \text { Na number of alleles, Ho observed heterozygosity, } \\
\text { He expected heterozygosity, } P I C \text { polymorphism information content, } P_{\text {HWE }} P \text {-values for Hardy-Weinberg equilibrium corrected } \\
\text { for multiple comparisons using the false discovery rate, } F(\text { Null) frequency of null alleles. }\end{array}$} \\
\hline
\end{tabular}

Based on the classification of Botstein et al [20], the average value of the polymorphic information content (PIC) was 0.839, ranging from 0.700 (MA-SSR-19) to 0.917 (MA-SSR-17), indicating that 20 loci were all highly informative $(P I C>0.500)$. These results indicated that there was a relatively high level of genetic diversity in these loci. Hardy-Weinberg equilibrium (HWE) analysis showed that eight loci (MA-SSR-09, MA-SSR-10, MA-SSR-11, MA-SSR-35, MA-SSR-38, MA-SSR-43, MA-SSR-51, and MA-SSR-58) significantly deviated from HWE $(P<0.05)$, and the remaining twelve loci were in accordance with HWE. These HWE deviations may be caused by natural selection, gene mutation, genetic drift, and the presence of null alleles. 


\section{Discussion}

With the application of NGS technology, genomics has developed rapidly in recent years, which provides an economical way to solve a wide range of questions. Especially, the K-mer method based on genome survey sequencing has been successfully applied for the estimation of genome size and genome structural information without prior knowledge of the non-model species. The genome size is closely associated with ploidy. As the increase in ploidy and chromosome numbers, genome size generally increases. Therefore, for polyploid organisms, the ploidy must be determined before detecting its genome information. $M$. anguillicaudatus has complex ploidy composition in the natural environment [21-23], such as diploid ( $2 \mathrm{~N}=50)$, triploid $(3 \mathrm{~N}=75)$, tetraploid $(4 \mathrm{~N}=100)$, pentaploid $(5 \mathrm{~N}=125)$, and hexaploid $(6 \mathrm{~N}=150)$. A previous study in natural populations of $M$. anguillicaudatus indicated that among Chinese $M$. anguillicaudatus populations, most individuals were diploid with $2 \mathrm{~N}=50$ chromosomes [12]. In this study, M. anguillicaudatus used for 2b-RAD sequence was diploid, which was identified by microsatellite markers. According to the reported genome data, except for few fish species [24-26], the genome size of most fish was generally less than $1 \mathrm{~Gb}$. The estimated genome size of diploid M. anguillicaudatus(1105.97 Mb)was larger than that in most fishes, such as Oryzias latipes (700.4 Mb) [27], Acanthogobius ommaturus (928.01 Mb) [28], and Hemibagrus wyckioides (728 Mb) [11], which was related to a higher number of repetitive sequences.

In addition to ploidy, many other factors including heterozygosity ratio, repeat content, whole genome duplication also affect the quality of a genome assembly. For genome assembly, if the heterozygosity rate is higher than $0.5 \%$, it is difficult to assemble, and if it is higher than $1 \%$, it is even more difficult [29]. The heterozygosity rate of $M$. anguillicaudatus was approximately $1.45 \%$, indicating a complex genome with the highest heterozygosity rate. Accordingly, we supposed that the low quality of assembled genome sequences with short scaffolds might be due to the high heterozygosity ratio of $M$. anguillicaudatus. Previous studies concluded sex determination type according to the differences in heterozygosity ratio between females and males [30, 31]. In $\mathrm{XX} / \mathrm{XY}$ sex chromosome system (male heterogamety), heterozygosity ratio in males was larger than that in females, therefore, female individuals used for $2 b$-rad sequencing may be more reasonable to ensure high-quality genome sequences. Besides heterozygosity rate, the GC content is one of the factors that directly affect sequence bias [32]. A high ( $>65 \%)$ or low (<25\%) GC content may cause sequence bias on the Illumina sequencing platform and further seriously affect genome assembly quality [33]. The average GC content of the $M$. anguillicaudatus genome was $38.03 \%$, which was in the acceptable range and did not have an effect on genome sequence quality. This is the first genome survey report of $M$. anguillicaudatus and the assembled genome sequences give a preliminary understanding of the genomic characteristics before large-scale genome sequencing. However, highquality genome sequences of $M$. anguillicaudatus should be generated by using PacBio and $\mathrm{Hi}-\mathrm{C}$ techniques.

Compared with conventional methods of microsatellite development, identifying the microsatellite from genome survey is not only time- and cost-effective but also affords a way of isolating a large deal of microsatellite markers spread over the entire genome. In this study, except for mononucleotide repeats, the dinucleotide repeat motifs were the most frequent motifs and CG/CG dinucleotide microsatellites were the least abundant dinucleotide SSR loci, this result was in agreement with other microsatellite repeats studies as described previously in Danio rerio, Oreochromis niloticus, and Oreochromis latipes [34]. This may be due to the methylation of cytosine into thymidine [35].

Whole genome sequencing data not only consists of nuclear genome sequences but also involves mitochondrial genome sequences [36]. In aquatic animals, Xu et al [31] have assembled the mitochondrial genomes based on the whole genome sequencing data of Platycephalus sp.1. Although, we failed to isolate complete the mitochondrial genome from genetic data because of the complex genome. We still suggested such a commercial method to acquire mitochondrial genome should be extensively applied in future genome survey studies. Previous studies in the mitochondrial genome have found that the $M$. anguillicaudatus was divided into two clades and considered a polyphyletic group. One clade and other genus Misgurnus species got together into a monophyletic group [37], while the other clade was clustered with the species of the genus Cobitis [38-40]. With respect that $M$. anguillicaudatus have two mtDNA lineages, Kitagawa et al [41] stated mtDNA introgression hypothesis: one corresponds to the individuals carrying introgressed mtDNA from the genus Cobitis, while another one corresponds to the relic of M. anguillicaudatus with non-introgressed mtDNA. In terms of the hypothesis, we supposed that the M. anguillicaudatus in this study is the "introgressed" mitochondrial type.

\section{Conclusion}


In conclusion, the first genome survey study of $M$. anguillicaudatus was performed based on whole-genome sequencing data, and genomic information, including genome size, heterozygosity ratio, GC content, and repeat sequence ratio, was assessed. These results indicated that the genome of $M$. anguillicaudatus was complex with high heterozygosity and repeated sequences. Genomewide microsatellite motifs and 20 polymorphic microsatellite loci in the 30 wild populations were identified. Moreover, the mitochondrial genome was described and phylogenetic analysis was presented to explore its taxonomic status. All of this genetic information will be valuable for further studies on genome size evolution, natural resource conservation, population genetic diversity, and molecular marker-assisted selective breeding in M. anguillicaudatus.

\section{Declarations}

\section{Acknowledgments}

This study was supported by China Agriculture Research System of MOF and MARA (CARS-46) and the National Key R\&D Program of China (No. 2018YFD0901201).

\section{Conflicts of Interest}

The authors declare no conflict of interest.

\section{Authors' contribution}

Conceived and designed the experiments: GC ML. Performed the experiments, analyzed the genetic data: GH. Contributed reagents/materials/analysis tools: JC CC MW ZL FG MY. Wrote the paper: GH ML. All authors read, revised, and approved the manuscript.

\section{Data Availability}

The data used to support the findings of this study are available from the article and its supplementary information.

\section{Ethics approval (Ethics)}

All experimental procedures for loach handling were approved by the Animal Care and Ethics Committee of the Pearl River Fisheries Research Institute, Chinese Academy of Fishery Sciences.

\section{Consent to Participate (Ethics)}

This study was conducted at Pearl River Fisheries Research Institute, Chinese Academy of Fishery Sciences. The institution and all authors agreed to participate in this research.

\section{Consent to Publish (Ethics)}

The Author confirms:

that the work described has not been published before;

that it is not under consideration for publication elsewhere;

that its publication has been approved by all co-authors if any;

that its publication has been approved explicitly by the responsible authorities at the institution where the work is carried out.

The authors agree to publication in the journal Molecular Biology Reports.

\section{References}


[1] Zhou W, Hu YY, Sui ZH, et al (2013) Genome survey sequencing and genetic background characterization of Gracilariopsis lemaneiformis (Rhodophyta) based on next-generation sequencing. PloS One 8(7): e69909.

[2] Lu X, Luan S, Kong J, et al (2017) Genome-wide mining, characterization, and development of microsatellite markers in Marsupenaeus japonicus by genome survey sequencing. Chin J Oceanol Limn 35(1): 203-214.

[3] Shi LL, Yi SK, Li YK (2018) Genome survey sequencing of red swamp crayfish Procambarus clarkii. Mol Biol Rep 45(5): 799-806.

[4] Song H, Zhang YX, Yang MJ, et al (2018) Genome survey on invasive veined rapa whelk (Rapana venosa) and development of microsatellite loci on large scale. J Genetics 97: e79-e86.

[5] Li ZY, Tian CX, Huang Y, et al (2019) A first insight into a draft genome of silver sillago (Sillago sihama) via genome survey sequencing. Animals 9(10): 1-8.

[6] Romana-Eguia MRR, Ikeda M, Basiao ZU, et al (2004) Genetic diversity in farmed Asian Nile and red hybrid tilapia stocks evaluated from microsatellite and mitochondrial DNA analysis. Aquaculture 236(1): 131-150.

[7] O'Connell M, Wright JM (1997) Microsatellite DNA in fishes. Rev Fish Biol Fisher 7(3): 331-363.

[8] Andersson L, Haley C, Ellegren H, et al (1994) Genetic mapping of quantitative trait loci for growth and fatness in pigs. Science 263(5154): 1771-1774.

[9] Fang S, Wu R, Shi X, et al (2020) Genome survey and identification of polymorphic microsatellites provide genomic information and molecular markers for the red crab Charybdis feriatus (Linnaeus, 1758) (Decapoda: Brachyura: Portunidae). J Crustacean Biol 40(1): 76-81.

[10] Qiu B, Fang S, Ikhwanuddin M, et al (2020) Genome survey and development of polymorphic microsatellite loci for Sillago sihama based on Illumina sequencing technology. Mol Biol Rep 47(4): 3011-3017.

[11] Zhou YL, Wu JJ, Wang ZW, et al (2021) Microsatellite polymorphism and genetic differentiation of different populations screened from genome survey sequencing in red-tail catfish (Hemibagrus wyckioides). Aquac Rep 19: 241-250.

[12] Zhong J, Yi S, Ma L, et al (2019) Evolution and phylogeography analysis of diploid and polyploid Misgurnus anguillicaudatus populations across China. Proc Royal Soc B 286(1901): 20190076.

[13] Li YJ, Yin J, Wang JB, et al (2008) A study on the distribution of polyploid loaches in China. Nippon Suisan Gakk 74(2): 177182.

[14] Huang SQ, Cao XJ, Tian XC, et al (2016) High-Throughput sequencing identifies microRNAs from posterior intestine of loach (Misgurnus anguillicaudatus) and their response to intestinal air-breathing inhibition. PloS One 11(2): e0149123.

[15] Feng B, Yi SV, Zhang MM, et al (2018) Development of novel EST-SSR markers for ploidy identification based on de novo transcriptome assembly for Misgurnus anguillicaudatus. PloS One 13(4): e0195829.

[16] Liu BH, Shi YJ, Yuan JY, et al (2013) Estimation of genomic characteristics by analyzing k-mer frequency in de novo genome projects.

[17] Luo RH, Liu BH, Xie YL, et al (2012) SOAPdenovo2: an empirically improved memory-efficient short-read de novo assembler. Gigascience 1(1): 30-30.

[18] Huelsenbeck JP, Ronquist F (2001) MRBAYES: Bayesian inference of phylogenetic trees. Bioinformatics 17(8): 754-755.

[19] Schuelke M (2000) An economic method for the fluorescent labeling of PCR fragments. Nat Biotechnol 18(2): 233-234.

[20] Botstein D, White RL, Skolnick M, et al (1980) Construction of a genetic linkage map in man using restriction fragment length polymorphism. Am J Hum Genet 32(3): 314-331. 
[21] Cui L, Abbas K, Yu Y, et al (2013) First record of the natural occurrence of pentaploid loach, Misgurnus anguillicaudatus in Hubei Province, China. Folia Zool 62(1): 14-18.

[22] Abbas K, Li MY, Wang WM, et al (2009) First record of the natural occurrence of hexaploid loach Misgurnus anguillicaudatus in Hubei Province, China [J]. J Fish Biol 75(2): 435-441.

[23] Arai K (2003) Genetics of the loach, Misgurnus anguillicaudatus: recent progress and perspective. Folia Biol 51: 107-117.

[24] Lien S, Koop BF, Sandve SR, et al (2016) The Atlantic salmon genome provides insights into rediploidization. Nature 533: 200205.

[25] Xu P, Zhang XF, Wang XM, et al (2014) Genome sequence and genetic diversity of the common carp, Cyprinus carpio. Nat Genet 46(11): 1212-1219.

[26] Berthelot C, Brunet F, Chalopin D, et al (2014) The rainbow trout genome provides novel insights into evolution after wholegenome duplication in vertebrates. Nature Communications 5(1): 3657-3667.

[27] Kasahara M, Naruse K, Sasaki S, et al (2007) The medaka draft genome and insights into vertebrate genome evolution. Nature 447(7145): 714-719.

[28] Chen BJ, Sun ZC, Lou FR, et al (2020) Genomic characteristics and profile of microsatellite primers for Acanthogobius ommaturus by genome survey sequencing. Bio Rep 40: BSR20201295.

[29] Marçais G, Kingsford C (2011) A fast, lock-free approach for efficient parallel counting of occurrences of k-mers.

Bioinformatics 27(6): 764-770.

[30] Huang YQ, Jiang DN, Li M, et al (2019) Genome Survey of Male and Female Spotted Scat (Scatophagus argus). Animals 9(12): $1-17$.

[31] Xu SY, Zhang H, Gao TX (2020) Comprehensive whole genome survey analyses of male and female brown-spotted flathead fish Platycephalus sp.1. Genomics 112(6): 4742-4748.

[32] Cheung M, Down T A, Latorre I, et al (2011) Systematic bias in high-throughput sequencing data and its correction by BEADS. Nucleic Acids Res 39(15): e103.

[33] Aird D, Ross MG, Chen WS, et al (2011) Analyzing and minimizing PCR amplification bias in Illumina sequencing libraries. Genome Biol 12(2): 1-14.

[34] Lei Y, Zhou Y, Price M, et al (2021) Genome-wide characterization of microsatellite DNA in fishes: survey and analysis of their abundance and frequency in genome-specific regions. BMC Genom 22(1): 421-421.

[35] Schorderet DF, Gartler SM (1992) Analysis of CpG suppression in methylated and nonmethylated species. PNAS 89(3): 957961.

[36] Ji D, Sun Z, Song N, et al (2020) The complete mitochondrial genome of Jaydia lineata (Perciformes, Apogonidae) obtained by next-generation sequencing. Mitochondrial DNA B 5(3): 2507-2508.

[37] He SP, Gu X, Mayden RL, et al (2008) Phylogenetic position of the enigmatic genus Psilorhynchus (Ostariophysi: Cypriniformes): Evidence from the mitochondrial genome. Mol Phylogenet Evol 47(1): 419-425.

[38] Cui Z, Zhou X, Yu Y, et al (2014) Comparative analysis of mitochondrial genomes in distinct nuclear ploidy loach Misgurnus anguillicaudatus and its implications for polyploidy evolution. PloS One 9(3): e92033.

[39] Zeng LG, Wang JH, Sheng JQ, et al (2012) Molecular characteristics of mitochondrial DNA and phylogenetic analysis of the loach (Misgurnus anguillicaudatus) from the Poyang Lake. Mitochondr DNA 23(3): 187-200. 
[40] Lee SY, Bang IC, Nam YK (2018) Complete mitochondrial genome of albino cyprinid loach, Misgurnus anguillicaudatus (Cypriniformes: Cobitidae). Conserv Genet Resour 10: 507-510.

[41] Kitagawa T, Fujii Y, Koizumi N (2011) Origin of the two major distinct mtDNA clades of the Japanese population of the oriental weather loach Misgurnus anguillicaudatus (Teleostei: Cobitidae). Folia Zool 60(4): 343-349.

\section{Figures}

A

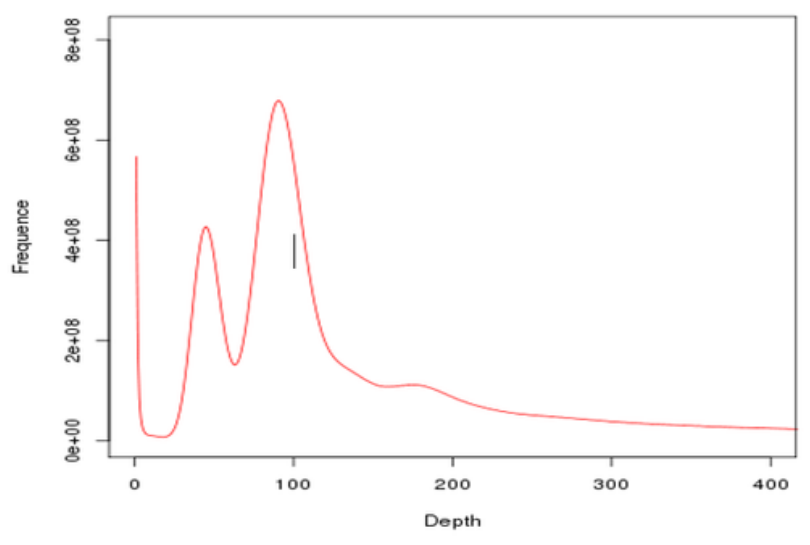

B

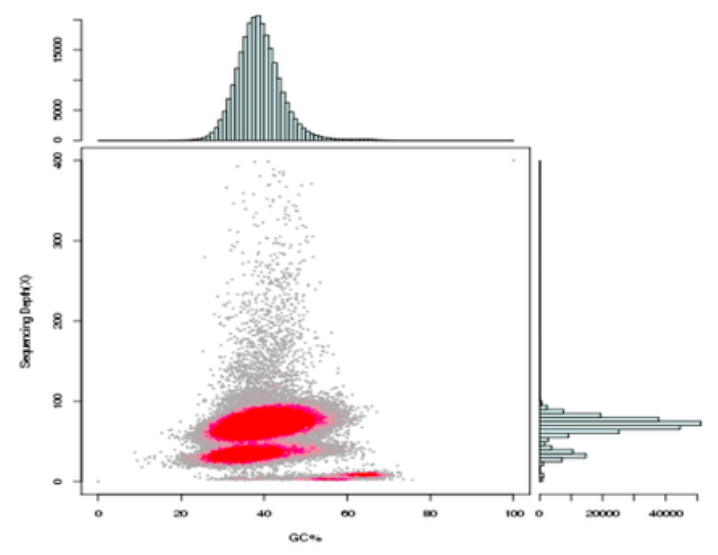

\section{Figure 1}

Results of genome survey using NGS. (A) K-mer ( $K=17)$ analysis for estimate genome size and heterozygote frequency of M. anguillicaudatus. The $\mathrm{x}$-axis is depth $(\mathrm{X})$; the $\mathrm{y}$-axis is the proportion that represents the frequency at that depth divided by the total frequency of all depths. (B) Guanine plus cytosine (GC) content and depth correlation analysis of $\mathrm{M}$. anguillicaudatus. The $\mathrm{x}$-axis represents the GC content and the $y$-axis is the sequencing depth. The distribution of sequence depth is on the right side, while the distribution of GC content is at the top.
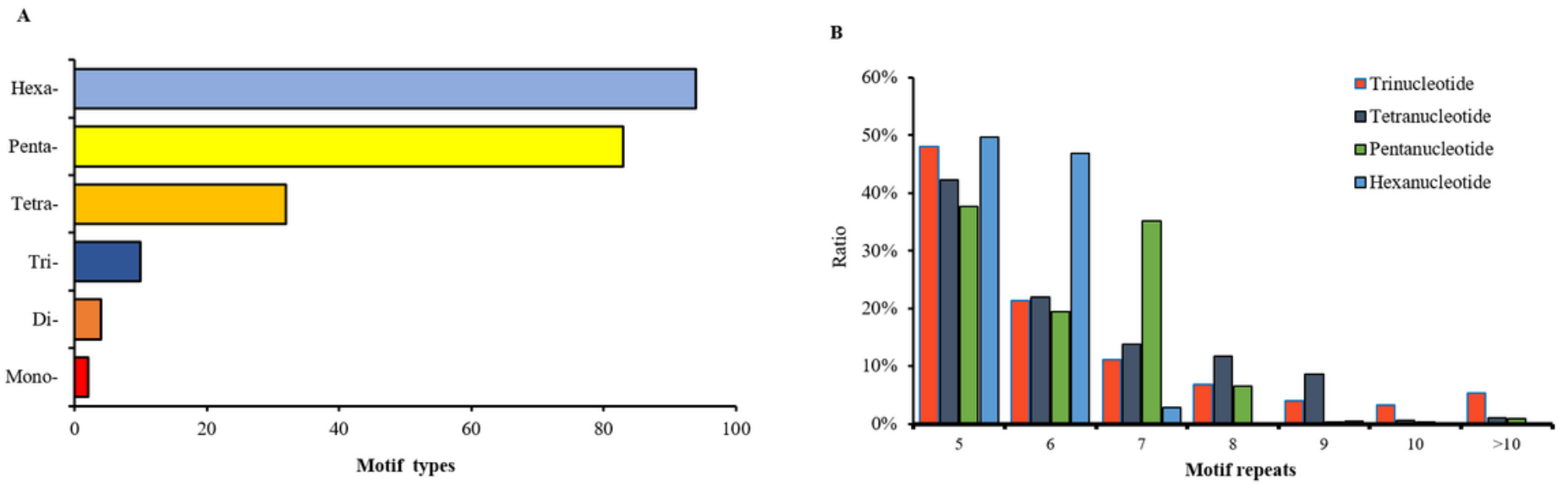

\section{Figure 2}

The motif types and frequency distribution of M. anguillicaudatus SSRs. (A) The motif types for mononucleotide, dinucleotide, tetranucleotide, pentanucleotide, and hexanucleotide. (B) Frequency distribution by the number of repeats for trinucleotide, tetranucleotide, pentanucleotide, and hexanucleotide. 
A

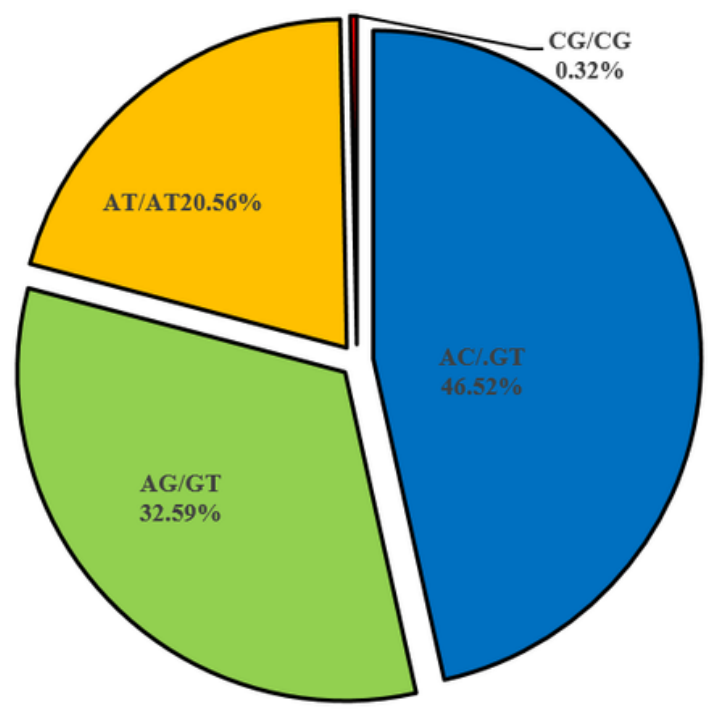

B

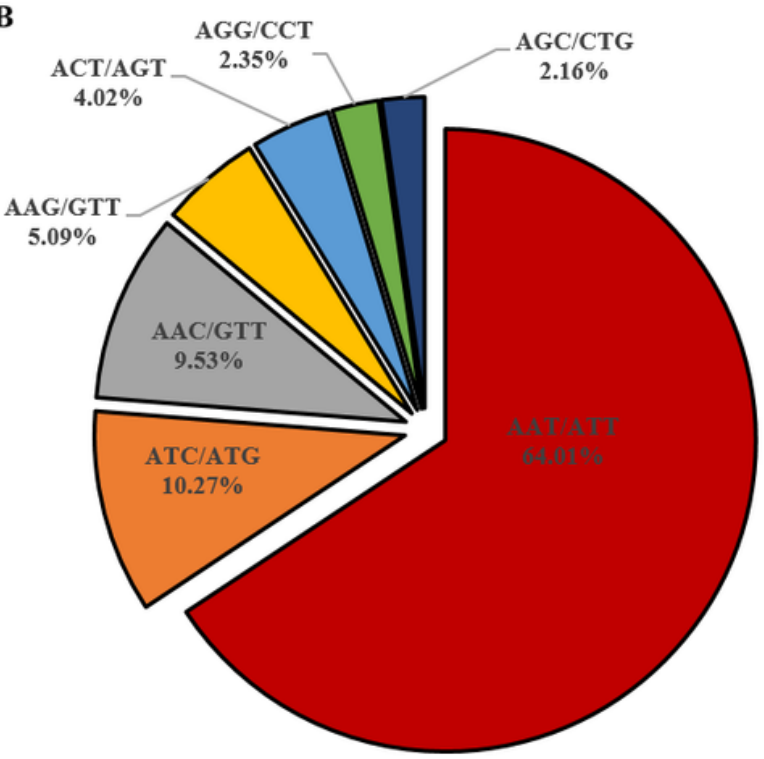

Figure 3

Frequency of identified microsatellite motif types in dinucleotide (A) and trinucleotide (B). 


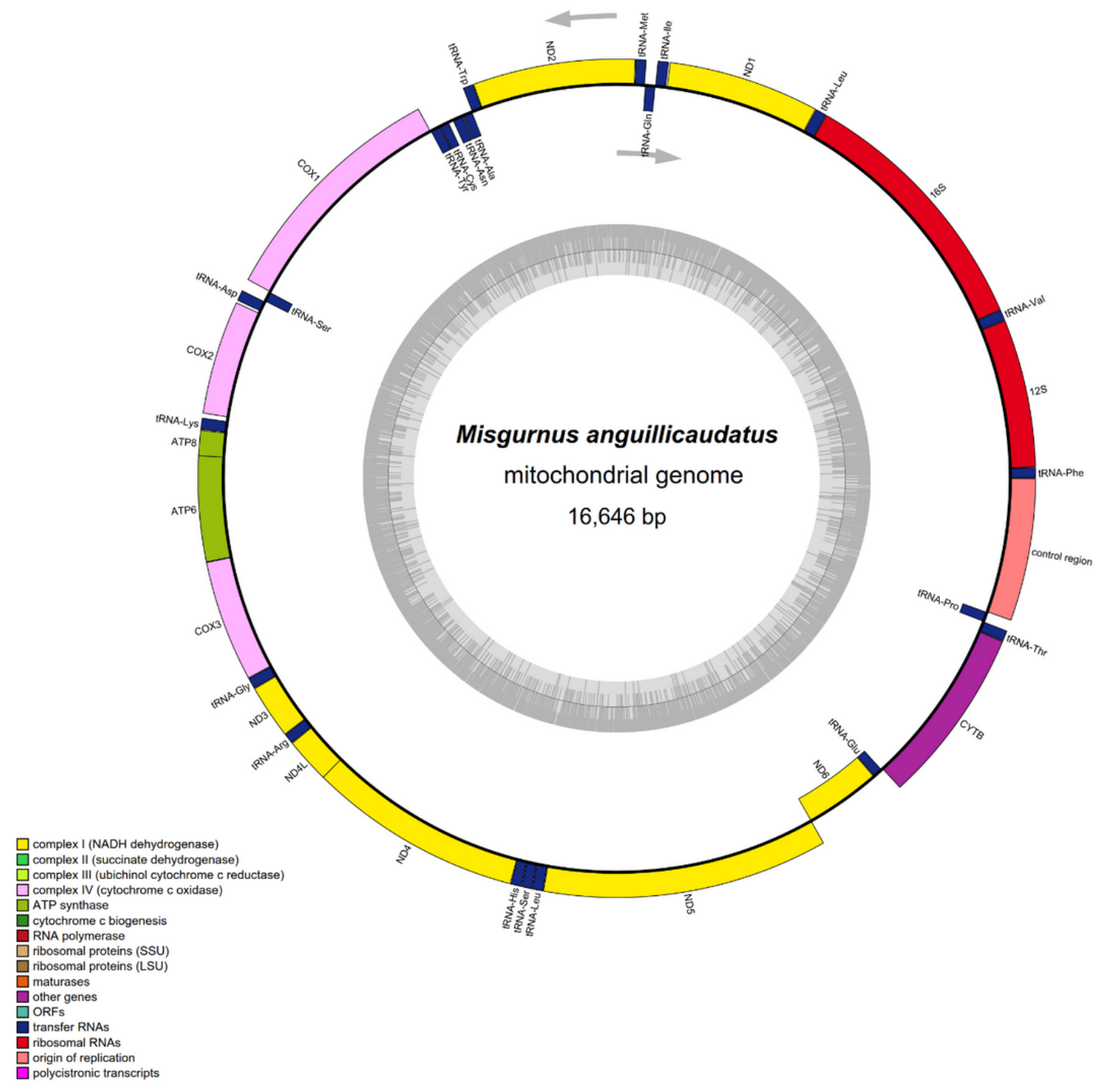

Figure 4

Gene map of diploid M. anguillicaudatus mitochondrial genome. 


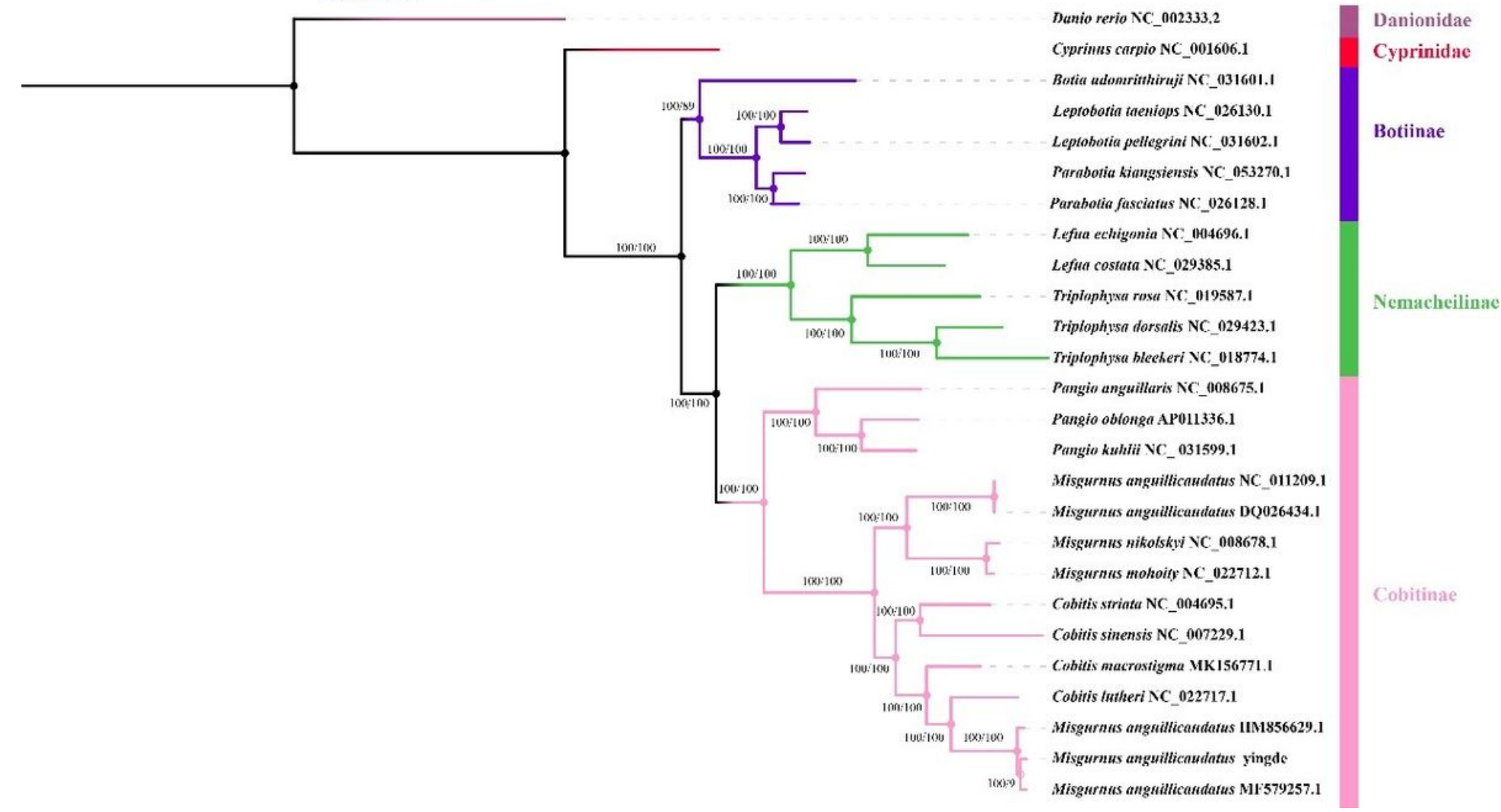

\section{Figure 5}

Phylogenetic analyses of diploid M. anguillicaudatus and other Cobitidae species. The species and their NCBI Accession NO. were listed in Supplementary material Table S5. The phylogenetic analyses were conducted based on the concatenated 13 mitochondrial protein-coding genes with Bayesian inference (BI) methods and maximum likelihood (ML). Numbers on the nodes represent support values inferred from BI (left) bootstrap and ML (right) probability analyses, respectively.

\section{Supplementary Files}

This is a list of supplementary files associated with this preprint. Click to download.

- Supplementarymaterial.docx 